\title{
Gestión del conocimiento e interacciones transformadoras de los actores gubernamentales para el desarrollo local
}

Castillo Arzola, Niria

Universidad de Ciego de Avila, Cuba niria@unica.cu

Garcés González, Roberto

Universidad Central de Las Villas, Cuba

rgarces@uclv.edu.cu

\author{
Santana Cruz, Julio Valentín \\ Universidad de Ciego de Ávila, Cuba
}

\section{Resumen}

La presente investigación se realizó a partir de la problemática referida a cómo las interacciones de los actores gubernamentales no están viabilizando el desarrollo local. En correspondencia con las características del municipio de Florencia, el trabajo se concibió y realizó desde una perspectiva comunitaria, que privilegió la participación como condición y estilo de trabajo. Este estudio, contribuyó al cambio en las interacciones de los actores gubernamentales y a someter a crítica la reproducción de prácticas que limitan el desarrollo. Se facilitó el tránsito de interacciones pasivas a transformadoras, porque posibilita articular creativamente las potencialidades endógenas del territorio con otras oportunidades internas o externas, teniendo como base los procesos de cooperación e implementación de proyectos. Al manifestarse la influencia de la gestión del conocimiento en las interacciones de los actores gubernamentales, los resultados obtenidos aportan a las Ciencias Sociológicas.
Palabras Clave: gestión del conocimiento; interacciones transformadoras; actor gubernamental; desarrollo local.

\section{Abstract}

The present investigation was made based on the problematic referred to how the interactions of the governmental actors are not making viable the local development. In correspondence with the characteristics of the municipality of Florence, the work was conceived and carried out from a community perspective, which privileged participation as a condition and style of work. This study contributed to the change in the interactions of the governmental actors and to criticize the reproduction of practices that limit development. The transition from passive to transformative interactions was facilitated, because it makes it possible to creatively articulate the endogenous potentialities of the territory with other internal or external opportunities, based on the cooperation and project implementation processes. When the influence of 
knowledge management on the interactions of government actors manifests itself, the results obtained contribute to the Sociological Sciences.

Keywords: knowledge management; transformative interactions; governmental actor; local development.

\section{INTRODUCCIÓN}

La presente investigación se sitúa en la dimensión local del desarrollo, teniendo como base la crítica a los hegemonismos discriminatorios $y$ excluyentes. Su realización está en consonancia con el contexto nacional e internacional, y posibilita asumir la realidad en sus mediaciones, referida a los actores sociales y sus interacciones, donde la gestión del conocimiento es el instrumento catalizador del carácter transformador de las mismas.

La importancia que reviste el estudio de las interacciones sociales ha sido reconocida desde los autores clásicos de la Sociología hasta la contemporaneidad, así se asevera en las obras de Blumer (1969), Shutz (1979) y Berger y Luckmann (2001). Son notorios sus aportes acerca de cómo las interacciones contribuyen a la transformación de la realidad a partir de los continuos reacomodos en función de las problemáticas surgidas.

Ahondar en el conocimiento de estas interacciones permite determinar aspectos esenciales de las relaciones entre actores, que privilegiando la simetría, posibilitará construir nuevas prácticas para el desarrollo local. Las interacciones están reproduciendo prácticas sociales aprehendidas por lo tanto se requiere que sean transformadoras, que lleven en sí la potencialidad de cambiar elementos de la realidad (Espina, 2010).

La producción científica elaborada desde la Red de Gestión Universitaria del Conocimiento para la Innovación y Desarrollo (GUCID) sistematizada en el libro Universidad, conocimiento, innovación y desarrollo local (Núñez et al., 2017), constituye un antecedente de esta investigación. Los artículos descritos allí contienen conceptos, metodologías y experiencias que se derivan del trabajo de la gestión del conocimiento para el desarrollo local. Los estudios mencionados, 
así como otros que les preceden, avalan que el modelo contexto/céntrico de producción del conocimiento es el adecuado en trabajos en que se analice este tipo de gestión para el desarrollo local (Núñez et al., 2017).

Esta investigación se guio por el objetivo de valorar la influencia de la gestión del conocimiento en las interacciones transformadoras de actores gubernamentales. Al aplicar la gestión del conocimiento a las interacciones transformadoras y efectuar análisis de los cambios en las interacciones transformadoras se obtuvieron los resultados que se exponen a continuación.

\section{MÉTODOS Y PROCEDIMIENTOS}

La población con la cual se trabajó fueron las 53 personas que pertenecen a la Asamblea Municipal del Poder Popular y al Consejo de la Administración Municipal de Florencia, provincia de Ciego de Ávila, Cuba. Se utilizaron cómo técnicas la entrevista en profundidad y los grupos de discusión.

\section{DESARROLLO}

Los sistemas gubernamentales, centralizados y verticalistas como el que se evidencia en la investigación, enseñan a ese individuo que luego deviene en actor a obedecer dócilmente los mandatos del otro. La superación de ese elemento pasivo puede darse con la preparación de los actores para que aprendan a negociar con los que les dan la orden. No es cambiar las estructuras, es lograr que cuando tengan una idea sean capaces de plantearla, argumentarla y defenderla. La gestión del conocimiento que se requiere tiene que posibilitar la construcción de vínculos simétricos en la consecución de un desarrollo local desde las potencialidades y peculiaridades de cada lugar.

La caracterización de las interacciones transformadoras de los actores gubernamentales, así como el análisis teórico realizado acerca de las potencialidades de la gestión del conocimiento basada en el modelo contexto/céntrico, posibilitó un diseño de gestión para influir sobre 
las interacciones, logrando que su carácter transformador se evidenciara en la realidad de un desarrollo local desde la perspectiva de lo comunitario. A continuación se presenta la secuencia organizada de las dimensiones identificadas.

\section{Identificación del}

conocimiento relevante

En esta fase se definen las necesidades que existen en materia de gestión del conocimiento, para lo cual se utiliza como instrumentos, el análisis de documentos, los grupos de discusión y el árbol de problemas. Después se estructuran en demandas, según las dimensiones del desarrollo, y se seleccionan las prioridades en las que se va a trabajar.

Los problemas priorizados de la dimensión económico -productiva son: las insuficiencias en la planificación y bajos rendimientos del sector agropecuario. Las necesidades de conocimiento a corto y largo plazos son, la gestión estratégica del desarrollo y la gestión de programas y proyectos.
Los problemas priorizados de la dimensión ambiental son: la deficiente gestión de residuales líquidos y sólidos, las deficiencias en el manejo de suelos y de las energías renovables. Las necesidades de conocimiento a corto y largo plazos son la gestión ambiental, tecnologías para la conservación y recuperación de suelos y sobre el cambio de la matriz energética para el desarrollo en el municipio.

Los problemas priorizados de la dimensión sociocultural se refieren a la escasa participación ciudadana en proyectos y los indicadores demográficos desfavorables. Las necesidades de conocimiento a corto y largo plazos se refieren al trabajo comunitario integrado.

Los problemas priorizados de la dimensión institucional son: las insuficientes capacidades para generar una gestión pública eficiente, y las necesidades de conocimiento a corto y largo plazos son, la comunicación institucional, gestión estratégica del desarrollo, gestión de programas y proyectos, gestión de planteamientos, trabajo comunitario 
integrado y tecnologías de la información y las comunicaciones en el trabajo gubernamental.

\section{Adquisición del conocimiento relevante}

Esta adquisición se hace a partir de las redes internas y externas del conocimiento y se crea un almacén, considerado como un repositorio de información en la intranet de la AMPP. Aunque la conformación del grupo gestor se hace desde el diseño del proceso, se decide explicar aquí el contenido del mismo porque juega el rol decisivo en la adquisición del conocimiento. Para el presente estudio se decide que el grupo gestor sea el mismo que acompaña al coordinador de la línea estratégica gestión del conocimiento para el desarrollo local, el que fue aprobado por el CAM según el Acuerdo 923.

Para lograr el funcionamiento óptimo del Grupo, sus integrantes deberían preocuparse por desarrollar habilidades muy precisas y necesarias a sus funciones, que les permitiría fortalecer los procesos comunicativos y de interacción. Entre las más apremiantes estarian: aprender a escuchar con atención, a observar, a analizar, a planificar, a organizar y a tomar decisiones racionales. Les sería muy útil también: aprender a negociar, a trabajar en equipo, a crear con originalidad y a emplear las nuevas tecnologías de la información.

Además deben tener nociones básicas sobre gestión de la información y del conocimiento, comunicación institucional, gestión documental y archivística, propiedad industrial, gestión ambiental, planeación estratégica y perfeccionamiento empresarial. Habrá expertos en estos temas dentro del Grupo, lo más importante es la disposición permanente a la preparación y autopreparación.

Los miembros del Grupo devienen en actores protagónicos en el accionar socio económico y político cultural del municipio. A los ojos de los demás sus actuaciones, en todos los órdenes de la vida, adquieren otra dimensión: la del representante gubernamental, la del dirigente político que, tiene que ser ejemplo y guía de un pueblo, que amén de muchísimas dejaciones y 
carencias, continúa siendo instruido, inteligente y muy correcto, y que sin llegar al entrometimiento soez, diariamente vigila, controla y examina sus desempeños. De ahí la importancia de aprender a comportarse teniendo en cuenta la flexibilidad, la comprensión, la tolerancia, el control emocional, la extroversión, la ecuanimidad, la perseverancia, el dinamismo, la seguridad, la precaución, la discreción y la motivación por la actividad.

Desarrollo de conocimientos relevantes

Para el desarrollo del conocimiento relevante se diseñan actividades y se utiliza la intranet que conecta a todos los actores que intervienen en el proceso de gestión del conocimiento. Actuará como punto de encuentro en la red, de forma tal que permita el intercambio y la cooperación entre todos los actores gubernamentales.

El desarrollo del conocimiento relevante se concreta en los productos y servicios nuevos o mejorados que se han podido implementar en el municipio.

- Servicios nuevos: diseño de proyectos de desarrollo.
- Servicios mejorados: aumento de la producción y distribución de dulces de harina, en conservas y de encurtidos de frutas y vegetales.

- Productos nuevos: Estrategia de Comunicación Institucional de la AMPP de Florencia.

- Productos mejorados: Metodología para la actualización de la estrategia de desarrollo local, Manual de procedimientos para la evaluación del desarrollo local y Metodología para la construcción de biodigestores de menos de $3 \mathrm{~m}^{3}$.

\section{Difusión del conocimiento relevante}

Para difundir el conocimiento relevante se utiliza la intranet, elaborada con ese fin, que posee un soporte tecnológico que visibiliza la Estrategia de Comunicación Institucional de la AMPP de Florencia. Los flujos comunicativos se producen tanto ascendentes como descendentes.

Los canales de comunicación a utilizar pueden ser directos e indirectos. Entre los primeros se destacan: Las reuniones, los eventos (congresos, fórum, talleres y exposiciones), las publicaciones internas, los buzones de sugerencias y 
las capacitaciones de cualquier tipo. Los canales indirectos a utilizar pueden ser internet, intranet, el portal informativo, la red interna, el teléfono y el correo electrónico.

El Modelo sociológico SECl (socialización, externalización, combinación e internalización), propuesto por Nonaka y Takeuchi (1999), es el que se utiliza en esta investigación para difundir el conocimiento relevante, pues su generalización, ya sea a través de las redes formales o informales, revierte tanta importancia como su propia generación:

"La conformación de las redes de innovación y de conocimiento debe formar parte de las estrategias de desarrollo en la agenda del gobierno local como procesos sistémicos que adquieren una expresión en la vida, a nivel individual, comunitario y local a partir de la cooperación, como elemento sustancial de lo comunitario". (Garcés, 2013: 76)

Una de las formas más comunes de socialización del conocimiento es la que se produce del movimiento del conocimiento tácito a tácito. En él la gestión del conocimiento presta atención y tiene en cuenta todas las potencialidades presentes, y sobre esa base propicia el encuentro de saberes. Las ferias expositivas de ganado menor y mayor que todos los años en el mes de agosto se realizan en Florencia, y que desde la edición del 2007 prevé un intercambio de experiencia entre los productores, han estimulado el aumento de la productividad de carne y leche en el municipio. En cuanto a la externalización, que es el cambio del conocimiento tácito a explícito, también se muestran resultados alentadores como por ejemplo, la confección de un sencillo Manual de buenas prácticas para la construcción y mantenimiento de biodigestores incluido una maqueta.

Otro modo de socializar los conocimientos relevantes es el denominado de combinación, que se produce cuando los saberes son explícitos. A esta forma corresponde la confección del Manual de procedimientos para medir el desarrollo local, uno de los productos mejorados obtenidos que fue elaborado tomando como referencia los indicadores propuestos en las escuelas de GUCID (Socorro et al, 2014). 
La internalización, constituye otra forma de socialización, y asegura el tránsito del conocimiento explícito al conocimiento tácito, es la meta superior en este empeño pues se garantizan los cambios de comportamientos con respecto a lo aprendido. En este tipo de socialización se enmarcan tres eventos científicos de carácter municipal que desde el 2015 han adquirido relevancia e intencionalidad, a saber: el Taller de ciencias sociales en la montaña, el evento FUMMA (Filial Universitaria Municipal y Medio Ambiente) y el Taller de usuarios de biodigestores. Estos encuentros sirven de plataforma de exposición, intercambio y difusión de las mejores experiencias en torno a las necesidades identificadas.

\section{Utilización del conocimiento relevante}

La utilización del conocimiento es una dimensión estrechamente vinculada a la del desarrollo del conocimiento, el producto o servicio que se obtiene en esta actividad tiene su concreción práctica en la utilización del conocimiento. La interacción de la gestión del conocimiento con los entornos claves a través de las estructuras de interfaces, debe tributar al uso adecuado e intensivo de las tecnologías existentes en diferentes disciplinas, además de contribuir al incremento de los estados financieros mediante la generación de proyectos que contribuyan a la conformación de nuevos o mejorados productos y servicios.

La ciencia y la tecnología le otorgan un valor agregado a la calidad de los productos, servicios y procesos. Este bien material obtenido logra una adecuada satisfacción de los consumidores influyendo positivamente en la calidad de vida de la sociedad florenciana como entorno social inmediato.

El diseño e implementación de proyectos constituye el indicador clave en la utilización del conocimiento para la solución a las necesidades identificadas en el primer paso de la gestión del conocimiento descrito anteriormente. Desde el 2015 y hasta enero del 2018 se han implementado proyectos que favorecen la transformación positiva de la realidad por parte de los actores gubernamentales. 
Las experiencias documentadas constituyen el núcleo para utilizar el conocimiento pues asegura su generalización y permite que se mantenga la lógica interna del proceso, es el insumo fundamental para lograr retener el conocimiento sin que dependa directamente de las personas que lo gestionan.

\section{Retención el conocimiento relevante}

Se utilizan insumos ya conseguidos en los pasos descritos anteriormente, así los repositorios de información obtenidos en el paso dos y las experiencias documentadas del anterior, se almacenan en bases de datos, catálogos, manuales de normas y procedimientos que se concretan en informes escritos 0 digitales, videos y grabaciones. Todo lo anterior forma parte de la documentación del proceso, y debe poseer las copias correspondientes para asegurarse la protección del conocimiento generado en y para el municipio.

Este paso está directamente vinculado a la apropiación social del conocimiento, como expresión de lo comunitario, que es la posición más consecuente ante las tendencias que inciden en el uso cada vez más privado del conocimiento, que es funcional a la dominación. La tendencia emancipadora debe estar dirigida a la socialización del conocimiento como fuente de poder compartido, porque:

(...) en las condiciones del municipio la forma más conveniente de retener el conocimiento es su socialización efectiva entre las personas y las organizaciones que lo necesitan ampliando su base social; por esa razón, los decisores locales deben privilegiar la importancia al trabajo en red estimulando espacios de socialización y diálogo de saberes (...). (Garcés, 2013: 75)

El grupo gestor es el encargado de seleccionar las experiencias documentadas a retener, hasta este momento todas las experiencias están dispuestas en la base de datos elaborada al efecto, debido al corto tiempo de la aplicación de la gestión del conocimiento y lo reducido del número de las mismas. Por lo tanto todas se encuentran almacenadas y la tarea fundamental de esta etapa es continuar con la actualización de las mismas. 
Se tiene implementado un sistema de vigilancia tecnológica que permite censar permanentemente el estado de las investigaciones y las soluciones de las necesidades identificadas en el primer paso. La FUM en el municipio es la encargada del mantenimiento del sistema.

Medición de la eficacia de la gestión del conocimiento

Para poder medir la eficacia, lo primordial es determinar el responsable de su implementación. En este estudio el máximo responsable es el Presidente de la AMPP de Florencia quien, debido a sus múltiples responsabilidades, delega estas funciones en el cuadro responsable de la línea tres de la Estrategia de Desarrollo Local denominada Gestión del Conocimiento para el Desarrollo Local. Este cuadro tiene la responsabilidad y la autoridad para asegurar que se establezcan, implementen, mantengan y desarrollen los procesos necesarios para el instrumento de gestión del conocimiento, además de informar al Presidente sobre el desempeño del mismo y de cualquier necesidad de mejora. Comprobará que la política y objetivos aprobados sean correctamente interpretados y aplicados.
Con la evaluación y el ajuste se determina el grado de cumplimiento de los objetivos propuestos, además de la identificación de los principales problemas e insatisfacciones que han salido a relucir durante el proceso. En esta etapa se realizan todas las modificaciones 0 correcciones necesarias para eliminar las deficiencias detectadas. Todo esto garantiza la mejora continua del proceso.

Se informa del seguimiento del sistema cuando el jefe de la línea de gestión del conocimiento rinde cuentas al CAM. Se realiza anualmente según cronograma establecido. Para asegurar el control del proceso se evalúa trimestralmente en el Grupo de Desarrollo Local.

Para cada objetivo se le designó un responsable y se verifica su cumplimiento según los plazos establecidos. Los resultados se explicitan en informes por cada responsable, se discute previamente en el seno del grupo gestor para presentarlos al Grupo de Desarrollo Local donde se aprueba y adoptan las medidas para corregir los problemas detectados. 
Las deficiencias detectadas son analizadas, haciendo énfasis en sus causas y efectos, después se elaboran las medidas preventivas y correctivas, las que se estructuran en las actividades de gestión del conocimiento.

\section{ANÁLISIS DE LA INFLUENCIA \\ DE LA GESTIÓN DEL \\ CONOCIMIENTO EN LAS \\ INTERACCIONES \\ TRANSFORMADORAS DE LOS ACTORES GUBERNAMENTALES \\ PARA EL DESARROLLO LOCAL DE FLORENCIA}

Se analizaron los resultados obtenidos de la aplicación de observaciones participantes y de los seis grupos de discusión en el primer semestre del año 2017, los datos se triangulan a partir de las dos variables en estudio, la gestión del conocimiento basada en el modelo contexto/céntrico y las interacciones transformadoras de los actores gubernamentales para el desarrollo local.
En la dimensión vínculos simétricos se evidencia que en los grupos de discusión, la influencia de las dimensiones desarrollar y difundir el conocimiento relevante, en sentido general, la preparación de los actores permitió que con sus argumentaciones quebraran la fórmula de ordeno y mando y dialogar para impulsar un proyecto, como es el caso del programa de equidad y género, el cual transverzaliza la gestión de la Estrategia de Desarrollo de Florencia.

Específicamente en los modos de relación hay cambios, pues aunque la forma interactiva continúe prevaleciendo, aparecen algunos conflictos desde la propia identificación de los conocimientos relevantes, sin embargo el consenso es el modo de relación emergente "si no nos ponemos de acuerdo es imposible determinar cuáles son las necesidades de conocimientos $y$ dónde y cómo las vamos a buscar"1.

1. Comentario que emergió del cuarto grupo de discusión. (N. de la A.) 


\section{En la intensidad de la relación} se observa en que los actores han sido capaces de ir paulatinamente evolucionando su posición y logran el intercambio de experiencias. Manifiestan que todo lo realizado en estos últimos años en materia de desarrollo local ha permitido tomar experiencias y aplicarlas en sus áreas de actuación. Aquí es clara la influencia de la difusión del conocimiento relevante, la tecnología social estrategia de desarrollo local ha permitido que la intensidad de la relación se acerque a las prácticas integradas.

La gestión del conocimiento ha tenido un impacto particular en la subdimensión intensidad de la relación, específicamente en el indicador de práctica generación de conocimientos, puesto que el inventario de innovadores a nivel municipal con un carácter menos formal y más en función de utilizar el conocimiento tácito para resolver problemáticas puntuales, ha traído como consecuencia la valorización del conocimiento como un bien in- tangible, pero con el cual se pueden crear bienes materiales. Los ejemplos más significativos redundan en el Programa de construcción de biodigestores y en el Proyecto de formación de capacidades para la disminución de la contaminación de las aguas por residuales líquidos en el poblado de Florencia, con los cuales se han podido resolver problemas relacionados con la contaminación ambiental y al mismo tiempo se ha logrado darle solución a planteamientos y quejas de la población.

Se plantea, por parte de los actores gubernamentales que existe un programa de gestión del conocimiento ${ }^{2}$ que ha permitido ir adquiriendo habilidades sobre la forma en que se puede gestionar el conocimiento, esto se expresa en lo grupos de discusión.

\section{La potenciación de iniciativas,} sí constituye un evento, dentro de la intensidad de la relación que se ha ido consolidando a partir del

2. La fundamentación del programa se plasma en la investigación Programa de gestión del conocimiento para el desarrolo local dirigido a la preparación de los miembros del Consejo de la Administración y directores de organizaciones laborales del municipio Florencia,Tesis de Maestría, tutorada en el 2014 por la autora principal de la presente investigación ( $\mathrm{N}$ de la $\mathrm{A}$ ) 
2015, por parte de la dirección de la Asamblea, esto se ha visto incentivado a partir de la aprobación de la ley N. 113 del Sistema tributario de fecha 23 de julio de 2012. Gaceta Oficial Ordinario N.53 de 21 de noviembre de 2012 que permite que el $1 \%$ de los sobrecumplimientos de todas las entidades radicadas en el municipios, puedan ser utilizadas para el desarrollo local, ello ha permitido que se generen proyectos que se puedan financiar desde el presupuesto municipal. El hecho que mediante la difusión del conocimiento relevante esa ley se haya socializado entre todos los actores gubernamentales ha permitido que en Florencia se puedan diseñar proyectos donde las fuentes de financiamiento sean diversas, no basta con leyes y decretos la preparación es clave.

Se ha determinado que en la reunión del grupo de desarrollo local, para su análisis y revisión, las propuestas de proyectos, las que una vez seleccionadas por este órgano serán llevados al CAM donde se determinará, según las prioridades establecidas, a que experiencia se le otorgará el respaldo financiero. Las prácticas integradas, que es el nivel superior de intensidad de la relación entre los actores gubernamentales aún no se pone de manifiesto.

Para la implementación de los proyectos, sobre todo los gestionados en los años 2015 y 2016, se necesita la aplicación de conocimientos, y que se concluya la construcción del conocimiento nuevo. Un ejemplo en tal sentido lo es el Proyecto fortalecimiento de capacidades para la producción local de materiales de la construcción, en la Unidad Empresarial de Base de Florencia. La implementación de este proyecto ha permitido combinar al conocimiento tácito, expresado en la tradición manufacturera de producir elementos de piso y de paredes, con la tecnología adquirida mediante un proyecto de colaboración. Se hace patente aquí la externalización del conocimiento y se evidencia cómo la dimensión de difusión del conocimiento influye en las interacciones, pues en procesos similares a este se logra que la experiencia sea documentada ${ }^{3}$.

3. Experiencia documentada por parte del grupo gestor de la gestión del conocimiento y que se recoge en un libro en proceso de edición, dirigido por especialistas de PRODEL ( $N$ de la A) 
Quedó demostrado que si ese conocimiento colectivo, combinatorio y local, se enfoca a resolver problemas sociales como el de la vivienda, propiciando la participación y las relaciones de horizontalidad, puede alcanzarse resultados sostenibles. En la actualidad el fondo habitacional de Florencia está considerado entre los mejores del país.

\section{DIMENSIÓN VISIONES ES-}

\section{TRATÉGICAS COMPARTIDAS}

En los miembros de la Asamblea se evidencian cambios en lo subjetivo, relacionados con las construcciones de la realidad, se denota una mejoría en la calidad de las intervenciones, disminuyen las frases estereotipadas y se evidencia una implicación personal, además son capaces de realizar críticas a las administraciones del municipio fundamentadas en elementos racionales. Se observa tanto en el CAM como en la AMPP se siente un sentido de pertenencia al municipio expresado en la elaboración de proyectos donde prima la relación cooperada, como es el caso de Barrio $X$ Barrio.

\section{Los procesos de negociación} han experimentado transformaciones, fundamentalmente en el seno del CAM. La gestión del conocimiento, demanda que los problemas se analicen desde perspectivas integradoras y que se vea la necesidad de utilizar conocimientos, tanto tácitos como explícitos y que se realicen procesos de investigación, atemperados a la dinámica de la gestión gubernamental. Esto se expresa en la necesidad de algunas mediaciones, como es el caso de la utilización del $1 \%$ y acuerdos de convivencia, aspecto que se observa relacionado con el Programa de equidad y género.

En la asamblea, la situación continúa siendo más desfavorable que en el CAM, sin embargo se evidencia una mejoría en las intervenciones, pues en los años 2015 y 2016 se realizaron un total de 342 intervenciones y de ellas 207 son de los delegados, también hay una mejoría en cuanto a las argumentaciones que se ofrecen.

\section{En la dimensión comunicación,} se evidencia como el proceso de gestión del conocimiento puesto en práctica ejerce influencia, puesto 
que se aplica la estrategia institucional de comunicación de la Asamblea Municipal del Poder Popular. Se destaca el aprendizaje y puesta en práctica de habilidades comunicativas para elaborar las respuestas que se le dan a los planteamientos. La totalidad de los actores gubernamentales continúan reconociendo la importancia de la comunicación, en un municipio como Florencia, que no posee ni radio, ni televisión, los talleres de comunicación cara a cara constituyen la mejor opción.

Otras formas emergentes de comunicación en el territorio lo son, la utilización de medios electrónicos y de videos realizados como parte de la estrategia de comunicación institucional, y puestos a disposición de la mayoría de los actores en el portal de la intranet del gobierno. Estas opciones constituyen uno de los medios construidos como aplicación, que soporta el proceso de gestión del conocimiento que se ha ido gestando entre los actores gubernamentales de Florencia.

\section{La dimensión de conocimien-} tos sobre interacción que tiene como indicador de práctica la subje- tividad comunitaria referida al desarrollo local presenta modificaciones. Los actores gubernamentales son capaces de plantear definiciones relacionadas con dimensiones del desarrollo, comunicación institucional, gestión gubernamental, gestión de programas y proyectos, y plantean la necesidad que se creen herramientas que permitan una gestión integral en la cual se integren los niveles municipales con los provinciales y los nacionales.

Las formas de pensar, la afectividad y los valores referidos a la interacción y al desarrollo local han sido modificadas, pues le otorgan mayor importancia a las interacciones informales, con ejemplos concretos de porque es así, sin demeritar el papel que juegan las formales. Aparece el reconocimiento que el desarrollo de Florencia se tiene que construir desde el municipio, y que no es similar al de otros municipios, provincias o al de la propia nación. Se evidencia la necesidad de discordar, y se tienen ideas, algunas puestas en prácticas de cómo lograr ese desarrollo, donde uno de los factores a tener en cuenta es el de gestionar el conocimiento. 
Los actores gubernamentales cooperan entre sí, proponen y diseñan proyectos, el ejemplo está en la Asamblea donde existen 12 proyectos de trabajo comunitario integrado que han surgido de las propias necesidades directas de la población, a partir de un diagnóstico participativo y donde los beneficiarios no solamente comparten espacio, sino que son los protagonistas del cambio requerido para el logro de la emancipación. Se puede afirmar que:

"Lo comunitario, como vínculo de simetría entre las personas, está presente en el desarrollo comunitario que devenga de una gestión municipal donde se pueda constatar la participación y cooperación individual y colectiva de las personas en torno a proyectos que son de autodesarrollo porque emergen como solución a las contradicciones esenciales de las que proceden sus malestares de vida cotidiana." (Alonso, 2011: 15)

De los resultados de las interacciones, específicamente de los devenidos de la aplicación del conocimiento y las tecnologías, destaca la propia Estrategia de Desarrollo Local convertida en un ins- trumento de gestión para el desarrollo que aglutina en sí a los demás instrumentos de planificación, es decir los programas que permiten concretar el momento táctico de la Estrategia donde resultan relevantes por su integración y diálogo de actores, los de materiales de la construcción, gestión del conocimiento y equidad y género.

Las características de interacciones transformadoras de los actores gubernamentales presentan puntos de contacto con los roles de la "persona facilitadora en el desarrollo territorial" (Costamagna y Larrea, 2017: 93), que son: crear espacios de diálogo, construir visión compartida, gestionar situaciones de conflicto, construir relaciones de confianza, construir agendas compartidas, conectar el territorio con las escuelas del pensamiento y los debates externos, conectar la teoría y la práctica, facilitar la reflexión y acción para construir capacidades colectivas en el territorio.

\section{CONCLUSIONES}

La gestión del conocimiento, basada en el modelo contexto/céntrico, encierra en sí misma una lógica in- 
terna que asegura su contribución a que las interacciones entre los actores gubernamentales transiten hacia lo trasformador. Posibilita la creación de espacios para la comunicación horizontal, potencia la cooperación y el reconocimiento del otro, en la búsqueda de soluciones a los problemas de la cotidianidad del municipio. Potencia la asunción de contradicciones y le permite a los actores gubernamentales transitar de lo reproductivo y acrítico de la vida cotidiana a posturas propositivas, desde argumentaciones sólidas, donde el reconocimiento de diferentes grados de conflictos se considera como necesario en el desarrollo.

El proceso de gestión del conocimiento explicitado emerge como dispositivo de cambio en tanto sea capaz de auto organizarse y corregirse en consonancia con las complejas condiciones de la gestión gubernamental en un territorio, en este caso un municipio. Las transformaciones que va a sufrir dependen de causalidades múltiples, azares e incertidumbres y de las innovaciones que el municipio, como célula del sistema político sea capaz de captar y generalizar o crear.
La influencia de la gestión del conocimiento, basada en el modelo contexto/céntrico, en las interacciones transformadoras entre los actores gubernamentales, se visualiza en el propio reconocimiento que la información y el conocimiento, como formas de poder, contribuyen a la democratización de sus accesos y a la de sus fuentes e instrumentos de producción, potenciando la creación de espacios interactivos de discusión y puesta en común, de donde surgen soluciones cada vez más complejas, y ajustadas al contexto en que se manifiestan, con argumentaciones lógicas construidas por los actores, para romper las reproducciones de verticalidad que se producen en las prácticas de dichos actores.

\section{BIBLIOGRAFÍA}

Alonso J., (2011) Municipio; entorno local para el desarrollo comunitario. Articulación de lo local y lo comunitario en el desarrollo comunitario. Santa Clara, editorial Feijóo.

Alonso, J., y D. Jara, (2016) "Participación diferenciada en proyectos de desarrollo". Simposio del Centro de Investigaciones Psicológicas y Sociológicas, en el marco de la Convención Internacional sobre Ciencia, Tecnología e Innovación [DC-Room], La Habana. 
Berger, P. L. y T. Luckmann, (2001) La construcción social de la realidad. Buenos Aires, Amorrortu.

Blumer, H., (1969) Symbolic Interactionism. Perpective and Method. New Jersey, Prentice Hall.

Castillo, N.; Santana, J. V. y M.R. González, (2017) "Impacto social de la gestión del conocimiento en el desarrollo municipal" en Trilogía y Sociedad [En línea] Volumen 9 No 16, enero 2017, Bogotá disponible en:

www.itmojs.itm.edu/index.php/trilogía/article/view/946 [Accesado el 21 de enero de 2017]

Costamagna, P. y L. Larrea, (2017) Actores facilitadores del desarrollo territorial. Una aproximación desde la construcción social publicaciones DEUSTO, Universidad de Deusto, Bibao, España

Espina, M., (2010) Desarrollo, desigualdad y políticas sociales. La Habana, Publicaciones Acuario.

Garcés, R., (2013) La gestión del conocimiento en las condiciones del municipio de Remedios como contribución a su desarrollo local. Tesis de doctorado. Universidad Central de las Villas "Marta Abreu", Cuba.

Nonaka, I., y H. Takeuchi, (1999) La organización creadora del conocimiento. México, OXFORD.

Nuñez, J.; Alcazar, A. T. y T. Proenza, (2017) "Una década de la red universitaria de gestión del conocimiento y la innovación para el desarrollo local en Cuba" en Retos de la dirección [En línea] año 11 número 2 enero 2017, Universidad de
Camagüey disponible en: http://revistas.reduc.edu.cu/index.php/retos/article/view/1946 [Accesado el 18 de enero de 2018]

Schutz, A., (1979) El problema de la realidad social. Buenos Aires, Amorrortu.

Socorro, A., Castro, N., y Y. Tartabull, (2014) "Línea base para la gestión municipal de la ciencia y la innovaciòn tecnológica en la provincia de Cienfuegos" en. Nuñez, J (comp.) Universidad, conocimiento, innovación y desarrollo local. (págs. 81-100). La Habana, Félix Varela. 